\title{
Biaxial tension on polymer in thermoforming range
}

\author{
S. Becker ${ }^{1}$, C. Combeaud ${ }^{1}$, F. Fournier ${ }^{1}$, J. Rodriguez, and N. Billon ${ }^{1, \text { a }}$ \\ ${ }^{1}$ Mines-ParisTech, CEMEF, UMR CNRS 7635, BP 20706904 Sophia Antipolis (France)
}

\begin{abstract}
This paper presents an experimental characterization of mechanical properties of a polyethylene terephtalate (PET) resin classically used in stretch blow moulding process. We have applied on such a material a well established experimental protocol at CEMEF, including new and relevant biaxial tensile tests. The experimental set-up relative to biaxial tension will be presented and described in a first part of the paper. Furthermore, we will focus on the experimental DMTA preliminary tests which are required to estimate the resin sensibility to temperature and strain rate in linear viscoelasticity domain. Finally, we will be interested in the material large strain behaviour: biaxial tensile results are presented and discussed. Finally, such an experimental approach should allow a relevant modelling of polymer physics and mechanics; this point will not be discussed here because of a lack of time.
\end{abstract}

\section{Scientific context}

Developing specific constitutive models for polymers appears of prime interest for the understanding of forming of packaging such as thermoformed or stretch blown parts. Among needed experimental methods, biaxial tension has became one of the most promising and relevant method allowing various loading state progress inside the material [1,2].

In parallel, strain field measurements demonstrated their relevance and accuracy in the domain of mechanical tests. Moreover, unloading and relaxation steps are known to be necessary to assess mechanical behaviour of materials. Finally, microstructure analysis, as a function of loading path, can also be an important point.

Consequently, a biaxial tensile machine combining all these facilities- various loading paths (uniaxial, plane, biaxial and equibixial tension) over a wide range of temperature up to draw ratio of 4 in both the two directions, combined with unloading or relaxation phases making it possible in situ strain field measurements and quenching of the sample- should be a very attractive machine.

In this paper, very first tests of such a device are presented. We study a PET resin usually used in stretch blow moulding process where significant biaxial solicitations mode is present. This characterization implies a better understanding of the PET mechanical behaviour involved in stretch blow moulding process. Then, optimising process conditions should be highly accelerated and improved by the use of numerical simulation, which implies a better modelling of polymer mechanics and physics.

\footnotetext{
a e-mail : Noëlle.Billon@mines-paristech.fr
} 


\section{Biaxial tension set-up}

\subsection{Sample geometry}

In this study, we use cross-shaped samples, as depicted on the Figure 1.

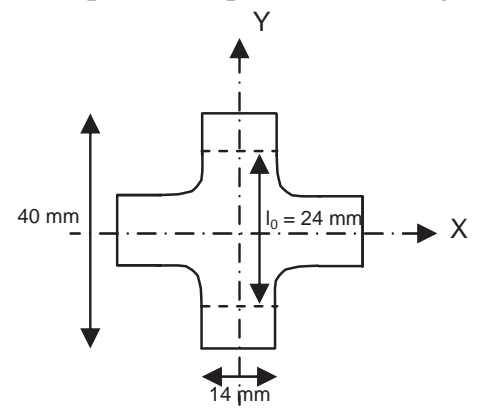

Fig. 1. Sample geometry used in biaxial tensile tests.

This cruciform specimen is maintained by four grips, thanks to which, either thin films $(\sim 100$ microns) or more massive devices ( 1 millimetre) can be stretched. The initial length $l_{0}$ between grips if of 24 millimetres (Figure 1).

\subsection{Experimental set-up}

Starting point is a machine, developed in the past by RHODIA and known as ETIFI ${ }^{\text {, a a a }}$ uniaxial, planar or sequential bi-axial tensile machine for films. The main characteristic of this machine is its ability of imposing efficient and controlled thermal cycles by the use of four ovens. At the present time, CEMEF owns the testing machine and has developed it so as to carry out more complex biaxial tensile tests.

Choice was made to adopt a system using four independent engines and specific samples (Figures 1 and 2).

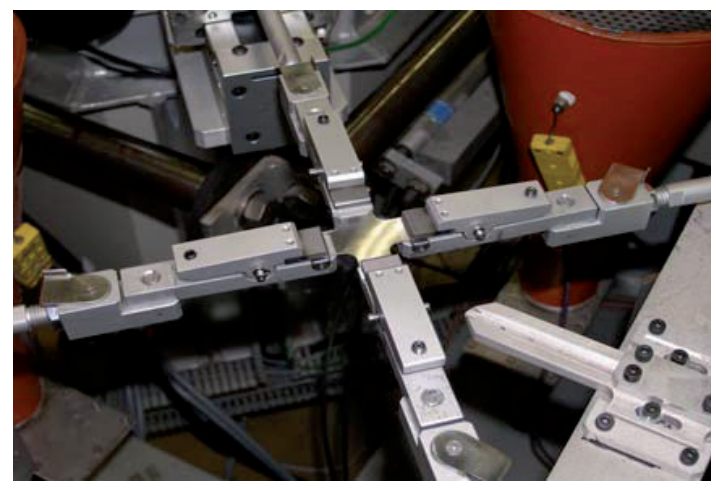

Fig. 2. Biaxial stretching system.

The grips displacements are hard driven by four current continuous motors controlled by servomotor amplifiers. The grips position and the load are respectively measured by LVDT and strain gauge sensors. Each arm is instrumented with one LVDT and one force sensor. Force sensors that are on the same tensile direction can be of different types, which allows accurate measurements from 
very low force (50 daN load sensor) up to high forces (500 daN load sensor) in case of strain hardening occurs. The maximal draw ratio is of 4 in both the two directions.

Also, we can impose either loadings, at constant cross head velocity, but also unloading or relaxation of the specimen. The figure 3 illustrates the tensile machine architecture.

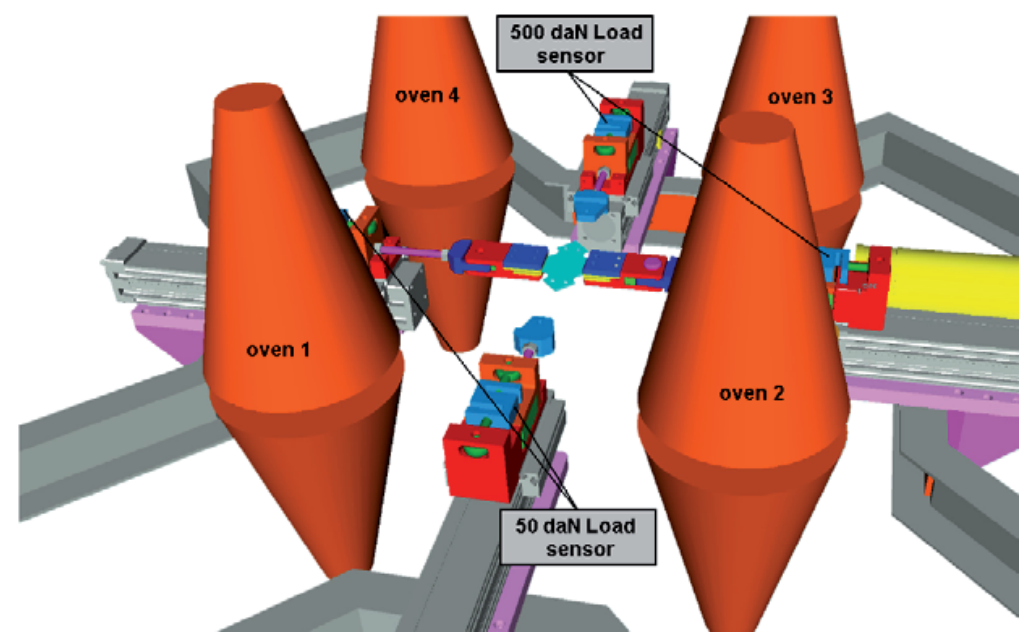

Fig. 3. Biaxial tensile machine.

Such a machine is able to combine tensile tests carried out over a wide range of temperature. The four air-pulsed ovens represented are able to move to the machine centre and to heat (or quench) the specimen during or after stretching. Indeed, the displacement of these ovens is imposed by four pneumatic cylinders allowing high speed thermal treatments on the sample under test. As a result, we are able to impose complex thermal cycles with a large temperature range varying from 20 to 200 ${ }^{\circ} \mathrm{C}$.

Computer test control and data acquisition are performed by a home-made software developed in LabVIEW $^{\circledR}$ graphical language.

\subsection{Measurement analysis}

During the tests, we measure, as a function of time, the displacement $\Delta l$ of each arm. Then the cumulated true strain can be approximated as follows :

$$
\varepsilon=\operatorname{Ln}\left(\frac{l}{l_{0}}\right)=\operatorname{Ln}\left(\frac{l_{0}+2 \times \Delta l}{l_{0}}\right)
$$

The local stress is estimated on the specimen diagonal $[2,3]$ :

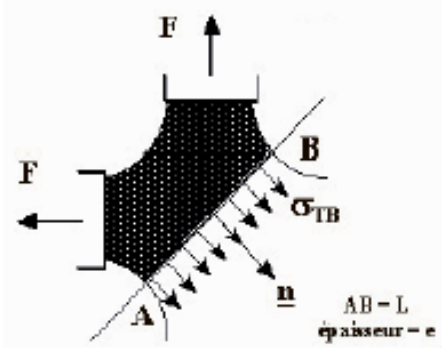

Fig. 4. Forces applied to the cruciform sample. 


$$
\sigma=\frac{\sqrt{2} F}{S(t)}=\frac{\sqrt{2} F}{e(t) \times L(t)}=\frac{\sqrt{2} F}{e_{0} \times L_{0} \times e^{-\varepsilon}}
$$

where $e_{0}$ and $\mathrm{L}_{o}$ are the initials thickness and diagonal length of the sample.

\section{Building up of experimental data base}

Our protocol to buid up the experimental data base is based on the use of time - temperature equivalence and of DMTA measurements. Our purpose is a correct description of behaviour on the rubbery plateau with a minimum number of tests.

Figure 5 depicts typical results for temperature scans at fixed frequencies.

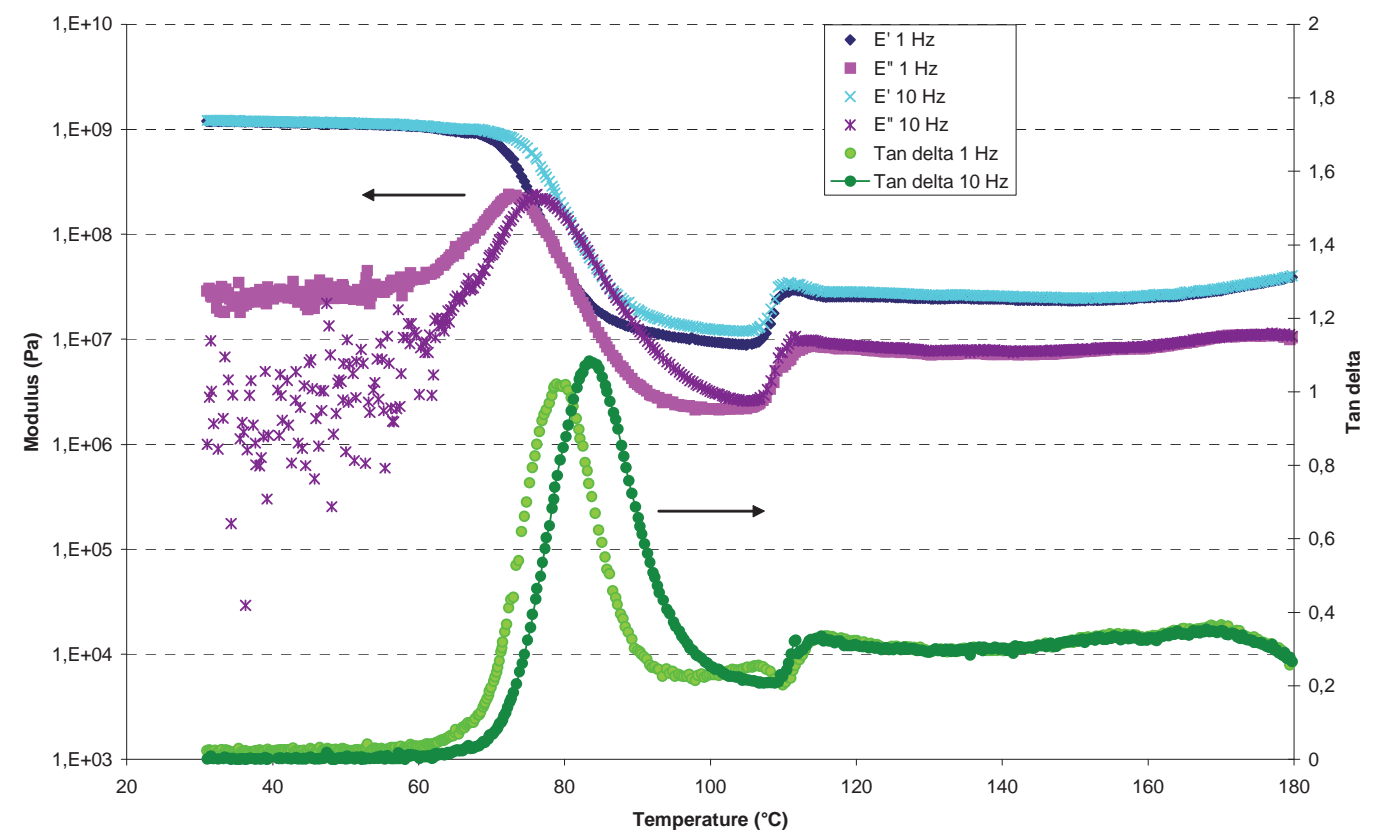

Fig. 5. Tan delta and modulus measurements of the PET resin, for a frequency of 1 and $10 \mathrm{~Hz}$, at a ramp temperature of $1{ }^{\circ} \mathrm{C} \cdot \mathrm{min}^{-1}$.

The alpha transition temperature, estimated at $1 \mathrm{~Hz}$ by this technique, is $80.4{ }^{\circ} \mathrm{C}$. This result is in agreement with many literature results $[4,5]$. The $\alpha$ transition is followed by another physical phenomenon which has to do with thermo-activated crystallisation. We identify a temperature of 108 ${ }^{\circ} \mathrm{C}$ at the beginning of this phenomenon where both modulus increase of half a decade.

Processing may take place from 80 to $110{ }^{\circ} \mathrm{C}$ at strain rates of several tens of s-1, the polymer has both lost its rigidity and has not yet thermo-crystallised. We have also to take care about the fact the frequency (or strain rate) may play an important role in the material response.

PET clearly obeys WLF law and a master curve can be deduced,e.g. for a reference temperature of $85^{\circ} \mathrm{C}$ (figure 6). 


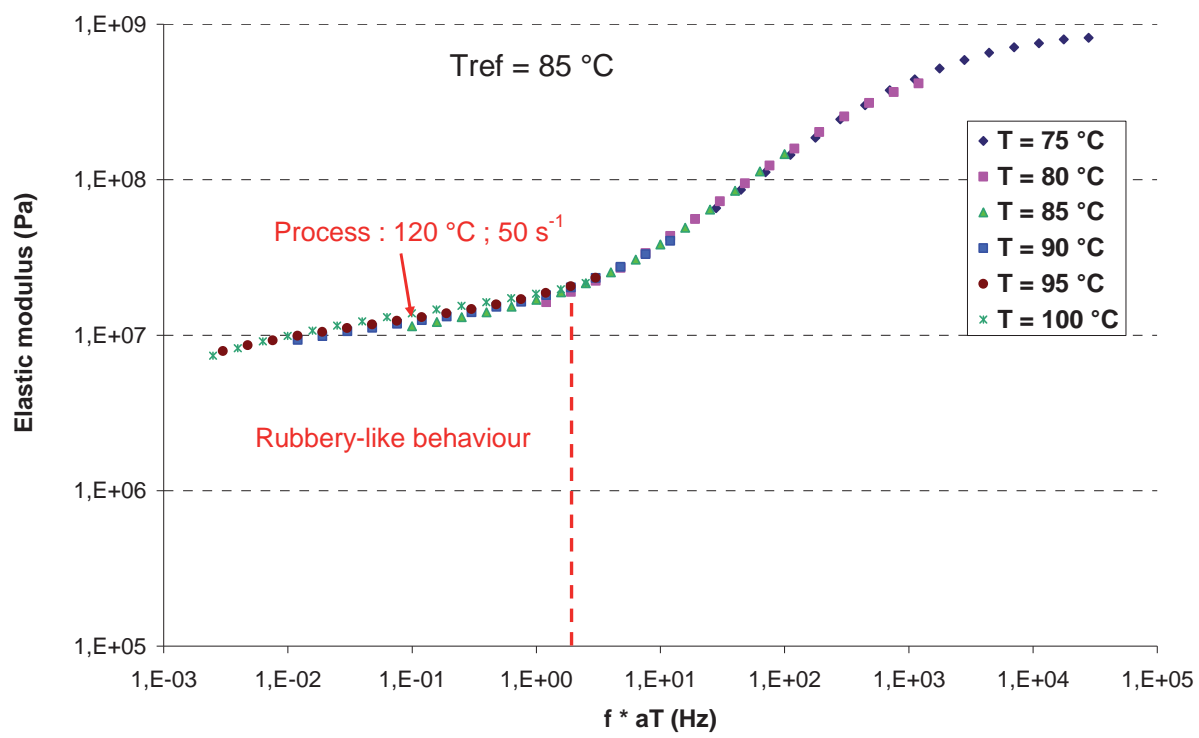

Fig. 6. Master curves of elastic modulus obtained at a reference temperature of $85^{\circ} \mathrm{C}$, for the PET resin.

From previous experiment we know that accurate state for PET during blowing are conditions were the equivalent strain rate at reference temperature ranges from 1 down to $0.01 \mathrm{~s}-1$. Experimental window will contain this range.

However as it is impossible to reach strain rate of a 100 on our device and due to thermal dissipation we should not use strain rates higher than 0.01. To make these experiments relevant they are performed at lower temperature according to WLF equivalence. For instance, a $120{ }^{\circ} \mathrm{C}-50 \mathrm{~s}^{-1}$, tension, characteristic of the window process, should be equivalent to a $85^{\circ} \mathrm{C}-0.2 \mathrm{~s}-1$ tests. This protocol is first validated in uni-axial tension.

\section{Mechanical behaviour at large strains: biaxial tensile tests}

\subsection{Biaxial tensile tests validation}

Preliminary tests were necessary to ensure that the biaxial test takes place in rigorous and optimal conditions. We carried out tests on PET samples, previously heated during 450 seconds at a temperature of $100{ }^{\circ}$ C. D.M.T.A. preliminary results had proved that cold crystallisation had not taken place yet for such heating durations, at $100{ }^{\circ} \mathrm{C}$. Then, still under the same temperature, a velocity $V$ of $0.1 \mathrm{~mm} \cdot \mathrm{s}^{-1}$ is imposed on each arm. The figure 7 compares the loading measurements issuing from each of the four arms. 


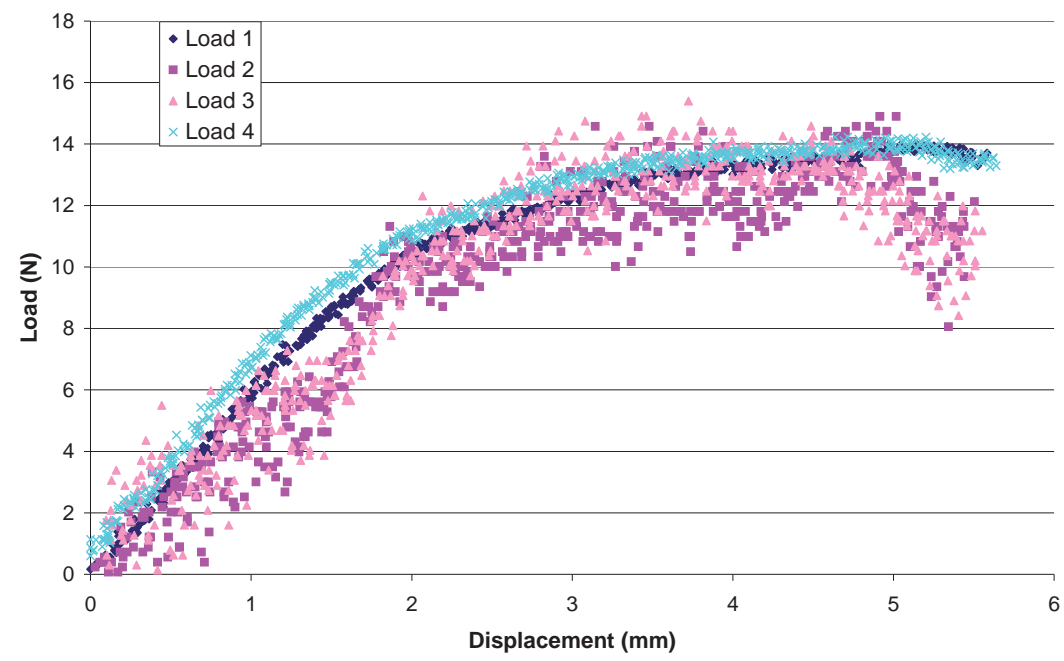

Fig. 7. Curves load/displacement issuing from each of the four arms, at a temperature of $100{ }^{\circ} \mathrm{C}$ and a arm velocity of $0.1 \mathrm{~mm} \cdot \mathrm{s}^{-1}$.

Through such experiments, we check that the loading history is well superimposed from an arm to another. It means that the equi-loading path imposed is equilibrated. Such a measurement is also in a good agreement with the stretched specimen obtained (Figure 8).

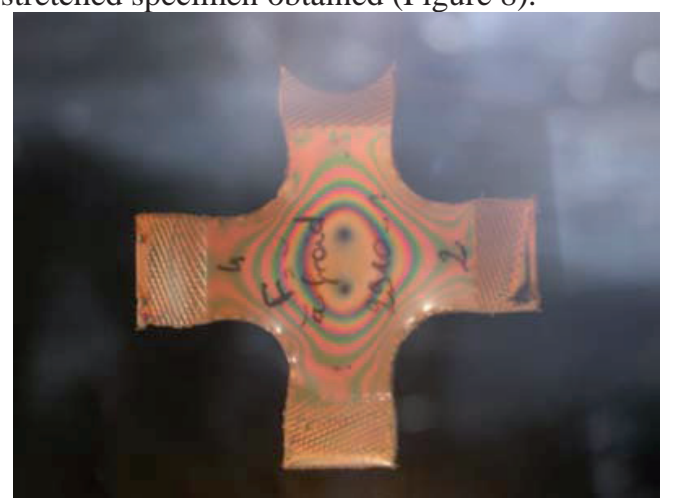

Fig. 8. Birefringence pattern observed on a PET specimen, equi-stretched, at a temperature of $100{ }^{\circ} \mathrm{C}$ and arms velocity of $0.1 \mathrm{~mm} \cdot \mathrm{s}^{-1}$, with an equivalent draw ratio of 1.9. The specimen has been ambient air cooled.

Birefringence pattern appears symmetrical with respect to the two main stretching directions. These results argue on the fact that the stretching system is well geometrically defined (perpendicularity of the two stretching directions, correct adjustment of the arms along the same stretching direction). The heating of the sample appears also homogeneous: local temperature measurement in front of the device and especially in the specimen centre has not put in evidence any pronounced thermal gradient.

Of course, the stress involved by the deformation imposed appears much more localized in the centre of the sample. The arms of the specimen are confronted to lower temperatures because of the grips presence and also as they are not strictly submitted to biaxial solicitations modes (uniaxial mode is much more present) : they are globally less deformed. In the future, using a painted speckle analyse should allow the measurement, during the stretching test, of the two-dimensional local strain field on the front of the specimen. 


\subsection{Experimental conditions}

We propose restrict measurements to monotonic tests, imposing the same controlled displacement velocity $V$ on the four arms; the loading remains equi-bi-axial. The stretching will be performed up to rupture of the sample.

Moreover, as defined thanks to D.M.T.A. results, we carried out tests for seven different sets of temperature/strain rate. The plan is summarised in the table 1 .

\begin{tabular}{|c|c|c|c|}
\hline Test number & Temperature $\left({ }^{\circ} \mathrm{C}\right)$ & Initial strain rate $\dot{\varepsilon}\left(\mathrm{s}^{-1}\right)$ & Equivalent strain rate $\left(\mathrm{s}^{-1}\right)$ \\
\hline 1 & 69 & $10^{-2}$ & 2331 \\
\hline 2 & 73 & $10^{-2}$ & 18 \\
\hline 3 & 78 & $10^{-2}$ & 0,35 \\
\hline 4 & 78 & $10^{-3}$ & 0,035 \\
\hline 5 & 83 & $10^{-3}$ & 0,0023 \\
\hline 6 & 83 & $5.10^{-4}$ & 0,0012 \\
\hline 7 & 96 & $10^{-2}$ & 0,00037 \\
\hline
\end{tabular}

Table 1. Sum-up of experimental conditions imposed during biaxial tensile tests.

We are not able to measure and impose a constant local strain rate during the test. Consequently, we impose, for each arm, a constant velocity displacement $V$ which leads us to an estimation of the initial strain rate defined by equation 4 .

$$
\dot{\varepsilon}=\frac{2 V}{l_{0}}
$$

with $l_{o}$ the initial length between grips.

\subsection{Biaxial tensile tests results}

Regarding preliminary biaxial tests accuracy, we are able to build up nominal stress/strain curves, for different strain rates and temperatures (Figure 9).

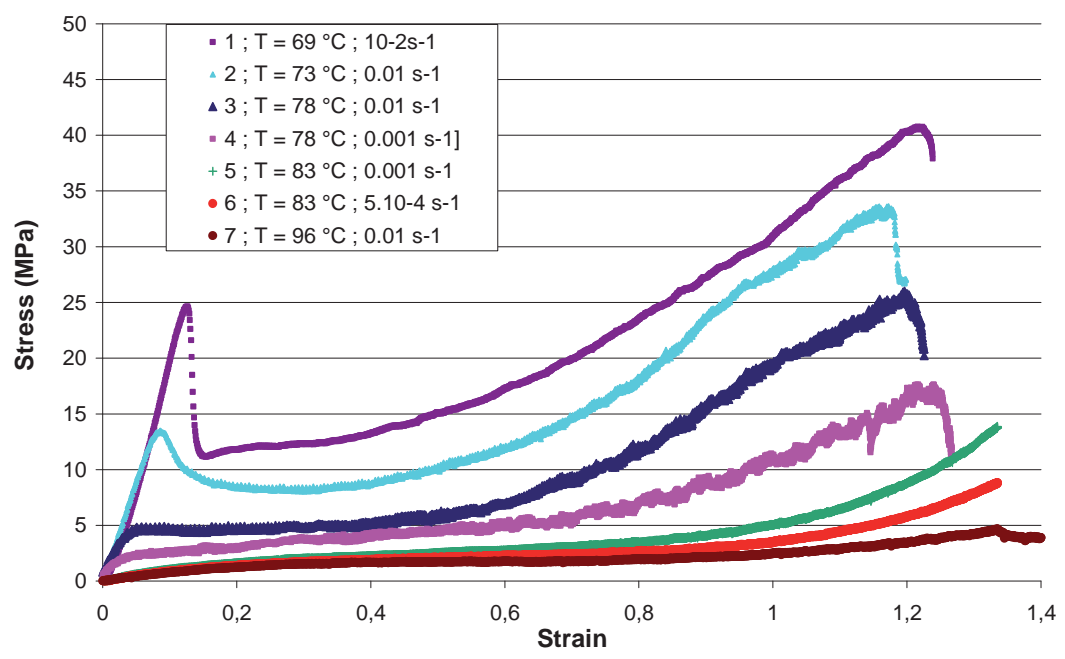

Fig. 9. Stress / Strain curves issuing from equi- bi-axial tensile tests on the PET resin, obtained at three different strain rates, for different temperatures. 
First of all, we can see that the mechanical response highly depends on strain rate as suggested by D.MT.A. This is not only a matter of parameters evolution but represents a drastic change in behaviour type itself, along the testing range: starting from a quasi glassy at low temperature (resp. high velocity) to a quasi fluid at high temperature (resp. low velocity) material. In between, a visco hyper elastic behaviour is observed that corresponds to processing range.

The experimental plan proposed allows us to observe these very different mechanical behaviours strongly sensitive to combined effects of temperature and velocity. These results appear crucial if we remind that during the blowing process itself, the material should experience very different conditions of temperature and velocity.

For lowest temperatures (test $\mathrm{n}^{\circ} 1$ or 2 ) coupled with highest strain rates, the behaviour appears glassy. For highest temperatures coupled (or not) with lowest strain rates, the mechanical behaviour at large strains is very similar to elastomers ones, representative of a hyper-elastic behaviour.

In both case, the strain-hardening phenomena, linked with material crystallisation, develops progressively with strain. For severe conditions (low temperature and high velocity), the phenomenon is all the earlier, getting faster well pronounced. This result is in agreement with [3].

What is also important to note towards such results is the influence of the parameter $a_{T} \times \dot{\varepsilon}$.

The tests $n^{\circ} 5,6$ and 7, for which the behaviour of the material appears very similar, are relative to the same value of $a_{T} \times \dot{\varepsilon}$ despite of different temperatures and strain rates. This validates our choice to control experimental conditions using equivalent strain rate at reference temperature. In addition, the combined effect of temperature and velocity summarised in this parameter should be the relevant physical tool for characterizing the mechanical behaviour of our resin, especially in biaxial tension conditions.

\section{Conclusions}

A PET resin has been characterized according to a rigorous protocol developed at CEMEF and extended to biaxial loadings. The first objective of such a study was to ensure that biaxial stretching could be carried out in optimal and controlled conditions, leading to a correct mechanical characterization of the material chosen. As a result, experimental data base confirm the complex behaviour of the PET resin as well as the great importance of strain hardening. We may imagine that the microstructure reorganisation involving crystallisation under deformation must be atypical in the case of biaxial loadings. What we may conclude today is that WLF like approach, identified with D.M.T.A analysis, appears to be a relevant tool, even in large strain domain and especially in biaxial tensile tests conditions. At a minimum, it was a pleasant approach to minimise and than optimise experimental protocol necessary for resin characterization. Indeed such a global protocol will reduce experimental effort needed for resin characterisation.

In the future, describing the resin response during a process as complex as blow moulding process should require pertinent modelling able to both represent the behaviour in uni and biaxial tensile tests.

\section{References}

1. S. Demmerle, J. P. Boehler, J. Mech. Phys. Solids, 41 (1), 143-181 (1993)

2. L. Chevalier, Y. Marco, Poly. Eng. Sciences, 42 (2), 280-298 (2002)

3. Y. Marco, Thèse de doctorat en Mécanique-Génie Mécanique-Génie Civil, ENS Cachan (2003)

4. E. Gorlier, Thèse de doctorat Sciences et Génie des Matériaux, ENSMP (2001)

5. M .Picard, Thèse de doctorat Sciences et Génie des Matériaux, ENSMP (2008) 